\title{
Molecular Viscosity Distribution in the Left Coronary Artery Tree
}

\author{
GD Giannoglou, *JV Soulis, TM Farmakis, GE Louridas \\ AHEPA University Hospital, Aristotle University of Thessaloniki, Greece \\ *Fluid Mechanics Division, Democrition University of Thrace, Xanthi, Greece
}

\begin{abstract}
The purpose of this study is to elucidate, probably for the first time, the distribution of the molecular viscosity in the entire Left Coronary Artery (LCA) tree. A three-dimensional computer model of the normal LCA tree, reported previously, was adopted for subsequent numerical analysis. The governing mass, momentum and energy flow equations were solved using a previously validated numerical (finiteelement analysis) code. The calculated results show high molecular viscosity regions appearing in zones opposite to all left coronary artery flow dividers on either of the two sides. These are regions where atherosclerotic plaques usually develop. Viscosity values change throughout the flow field. The distribution of high molecular viscosity along the walls is in agreement with the prone to atherosclerosis regions
\end{abstract}

\section{Introduction}

The role of blood viscosity in the development and progression of coronary artery disease is very important $[1]$, [2]. Moreover, according to non-Newtonian behavior, blood viscosity varies along the course of the vessel. This variation, depending on the vascular geometry and flow particularities, seems to play an important role in atherogenesis. However, little research has been focused on the exact role of the local viscosity differentiation. The purpose of this study is to elucidate, probably for the first time, the distribution of the molecular viscosity in the entire Left Coronary Artery (LCA) tree.

\section{Methods}

Experimental measurements of the intrathoracic spatial location of specified coronary segments on the normal human heart were previously reported [3], [4]. These studies were based on 83 angiographies taken from normal subjects. The exact intrathoracic location and course of each one of the 23 arterial segments and branches, which are commonly used by the physician to describe the localization of coronary disease, were reported. All data were processed with a Computing Aided Design (CAD) program, resulting into a threedimensional geometry model of the Left Coronary Artery (LCA) tree. This model included the LMCA, the LAD, the LCxA and their main branches: the first obtuse marginal (OM1), the second obtuse marginal (OM2), the first septal (S1), the first diagonal (D1), the second septal (S2), the second diagonal (D2), the third septal (S3), and the third diagonal (D3) branch. Figure 1 shows the geometry of the LCA tree, as it was above described.

Data describe the vessel centerline location (polar coordinates) and vessel diameter at each segment. In particular, the vessel centerline constructed using: a) radius, b) azimuth angle between projection of radius onto transverse plane and anterior-posterior axis and c) angle between radius and transverse plane. Once the vessel centerline construction is completed, the appropriate vessel diameter was applied at each segment, and the final vessel geometry was acquired via a computerized extrusion process of the pre-processing CAD program. These data were proven to be good enough to describe the exact anatomic location of the various anatomical segments and the final geometry was found to be satisfactory as a model simulating the normal human left coronary anatomy.

The lumen diameter of the LMCA orifice measured $4.5 \mathrm{~mm}$ while the corresponding values of the LAD and LCxA measured 3.7 and $3.4 \mathrm{~mm}$, respectively. The outlet LAD diameter at the apex of the heart was of 0.9 $\mathrm{mm}$, while the corresponding diameter of the LCxA at the outlet was of $1.3 \mathrm{~mm}$. For the first, second and third diagonal the corresponding diameters were of $1.1,1.0$ and $0.9 \mathrm{~mm}$, respectively, while for the first, second and third septal diameters were of $0.9,0.7$ and $0.7 \mathrm{~mm}$, respectively. For the LCxA branches, the outlet diameters of the first and second obtuse marginal were of 1.1 and $1.0 \mathrm{~mm}$.

All geometrical data were input into a specialized pre-processing program for grid generation. In total 44,452 grid nodes were utilized giving rise to 196,902 computational tetrahedral. Figure 2 shows details of the utilized non-structured grid of the human LCA tree. Figure 3 shows the distribution of computational grid nodes of the LAD vessel in a cross-sectional area 
located in region close to the origin of the second septal branch. Note the dense grid node distribution, for adequate depiction of physical blood quantities, in the near to the perimeter (endothelium) region. The relative error in the velocity components and in the haemodynamic parameter of wall shear stress was computed and used as a quantitative measure of the effect of grid density. The used mesh was based on the computational results of mesh-independence studies. The number of nodes used to define the mesh independence increased initially to 59524 nodes and at a later stage to 80889 nodes.

The blood velocity is assumed to be uniform at the orifice of LMCA. The applied inflow mimics typical coronary blood flow under resting conditions, $0.17 \mathrm{~m} / \mathrm{s}$. Flow discharges were set analogous to the third power of the branching vessel diameter (Murry's law).

All computational grid data, as well as all physical flow data determined from the boundary conditions, were imported into the main Computational Fluid Dynamics solver (Fluent release 5.5, FLUENT Inc.). The numerical code, which was previously validated, solves the governing Navier-Stokes flow equations. In the generality, these equations solve the mass, momenta and energy conservation. The assumptions made about the nature of the flow are that it is three-dimensional, steady, laminar, isothermal, with no external forces applied on it while the arterial wall is comprised from non-elastic and impermeable material. In their generality the flow governing equations are,

$$
\frac{\partial \rho}{\partial t}+\frac{\partial}{\partial x_{i}}\left(\rho u_{i}\right)=S_{m}
$$

where $\rho\left(\mathrm{kg} / \mathrm{m}^{3}\right)$ is the density, $t(\mathrm{sec})$ is the time, $u_{i}$ $(\mathrm{m} / \mathrm{s})$ are the velocity components along the $x_{i}(\mathrm{~m})$ axes, while $S_{m}$ is the added or subtracted mass of fluid in the flow field. The conservation of momentum along the $i$ direction is written,

$$
\frac{\partial}{\partial t}\left(\rho u_{i}\right)+\frac{\partial}{\partial x_{j}}\left(\rho u_{i} u_{j}\right)=-\frac{\partial p}{\partial x_{i}}+\frac{\partial \tau_{i j}}{\partial x_{j}}+\rho g_{i}+F_{i}
$$

Here, $p\left(\mathrm{~N} / \mathrm{m}^{2}\right)$ is the static pressure, $\tau_{i j}\left(\mathrm{~N} / \mathrm{m}^{2}\right)$ is the shear stress tensor, $\rho g_{i}\left(\mathrm{~N} / \mathrm{m}^{3}\right)$ and $F_{i}\left(\mathrm{~N} / \mathrm{m}^{3}\right)$ is gravity and externally acting forces, respectively. The blood was considered to be non-Newtonian fluid obeying to the exponential law [5]. According to this law the WSS, denoted by $\tau\left(\mathrm{N} / \mathrm{m}^{2}\right)$, is calculated as,

$$
\tau=[\eta(\dot{S})] \dot{S} \quad \eta(\dot{S})=k e^{\frac{T_{o}}{T}} \dot{S}^{n-1} \quad \dot{S}=\frac{\partial u_{i}}{\partial x_{j}}+\frac{\partial u_{j}}{\partial x_{i}}
$$

where $k, n$ are solution parameters, while $T(\mathrm{~K})$ and $T_{o}$ (K) are local temperature and reference temperature, respectively. For a typical satisfactory convergence solution a total of 600 time steps were required. Convergence was achieved when all velocity component changes from iteration to iteration were less than $10^{-6}$.

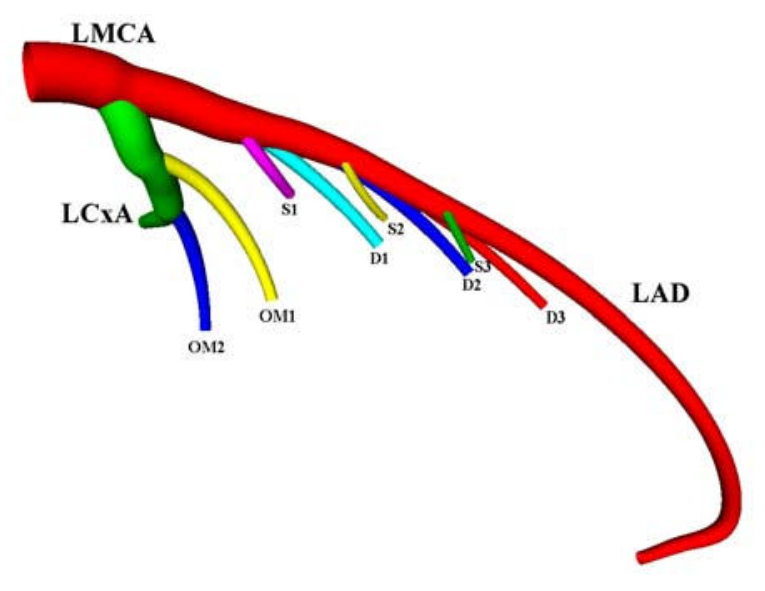

Figure 1. Normal LCA tree geometry.

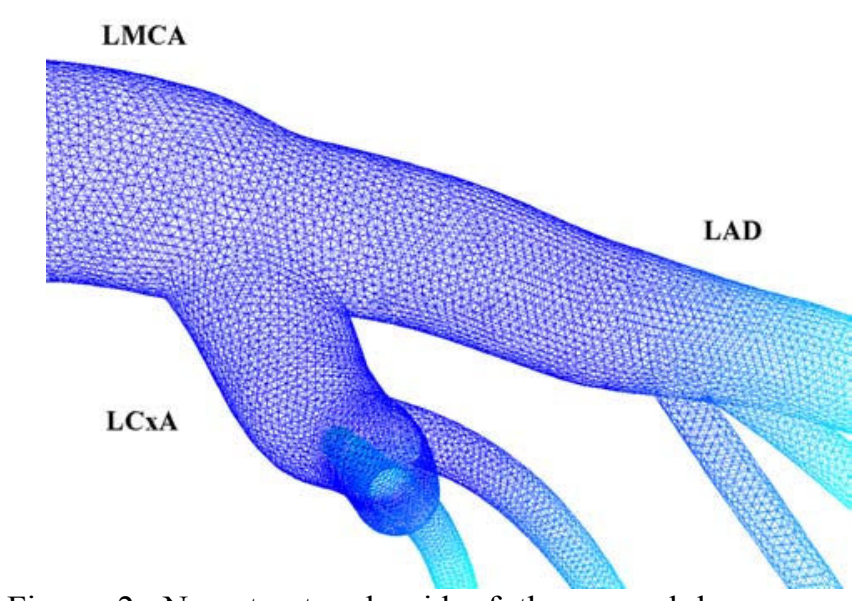

Figure 2. Non-structured grid of the normal human LCA tree used for computational analysis. Details of proximal tree segments.

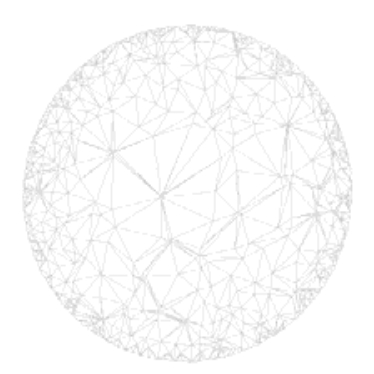

Figure 3. Grid details of a typical segment cross-section 


\section{Results}

All molecular viscosity values are shown in filled contours coupled with iso-contour line form. Contour labels appear in all figures, ranging from 1 to 15 , and correspond to the 15 color levels also shown in figures. Cut-off filters have been applied in all shown molecular viscosity values to adequately resolve the contouring distribution. Figure 4 shows the molecular viscosity (kg/m-s) magnitude distribution at the proximal Left Anterior Descending (LAD) branch. Figure 5 shows the contour plots of the molecular viscosity $(\mathrm{kg} / \mathrm{m}-\mathrm{s})$ magnitude distribution at the origin of the first diagonal (D1) branch, while Figure 6 shows the contour plots of the molecular viscosity (kg/m-s) magnitude distribution at the origins of the second (D2) and third diagonal (D3) branches. At distal LAD segment, Figure 7, low molecular viscosity prevails. Finally, Figure 8 shows the molecular viscosity distribution at an exit of the computational domain referring to a typical diagonal branch. Note the increased molecular viscosity values near to the crosssectional center.

The calculated results show high molecular viscosity regions appearing in zones opposite to all left coronary artery flow dividers on either of the two sides. These are regions where atherosclerotic plaques usually develop [6], [7]. Viscosity values change throughout the flow field. At proximal LAD regions the molecular viscosity values are of the order $0.007852 \mathrm{~kg} / \mathrm{m}-\mathrm{s}$, while at distal segments the corresponding viscosity values are $0.004738 \mathrm{~kg} / \mathrm{m}$-s.

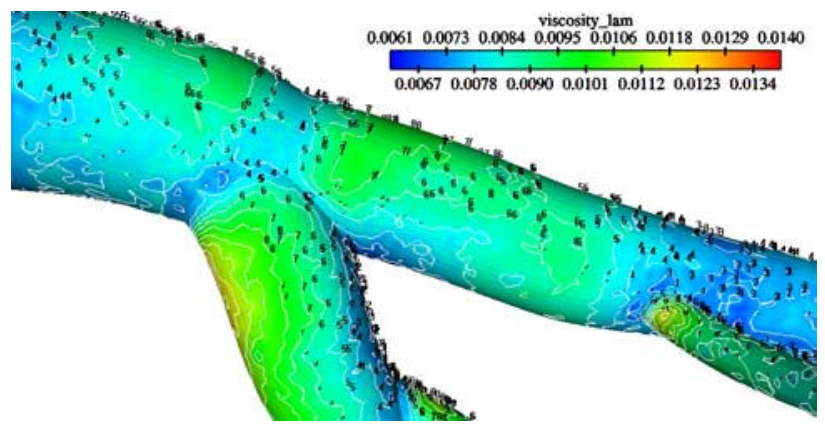

Figure 4. Contour plots of the molecular viscosity $(\mathrm{kg} / \mathrm{m}-\mathrm{s})$ magnitude distribution at the proximal Left Anterior Descending (LAD) branch. Contour labels range from 1 to 15 and correspond to the 15 corol levels shown in the bar.

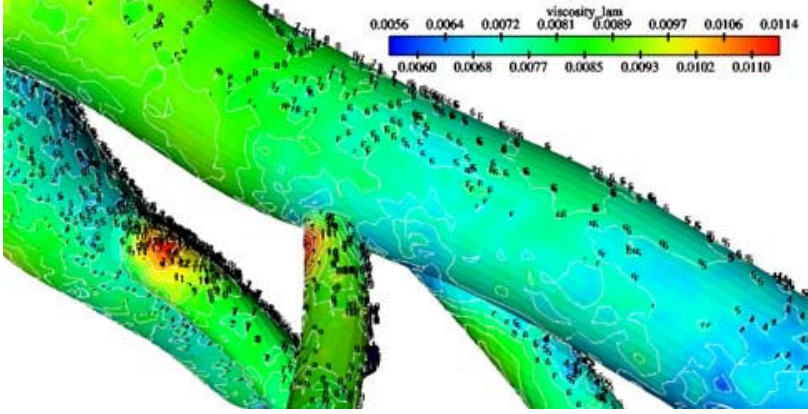

Figure 5. Contour plots of the molecular viscosity $(\mathrm{kg} / \mathrm{m}-\mathrm{s})$ magnitude distribution at the origin of the first diagonal (D1) branch.

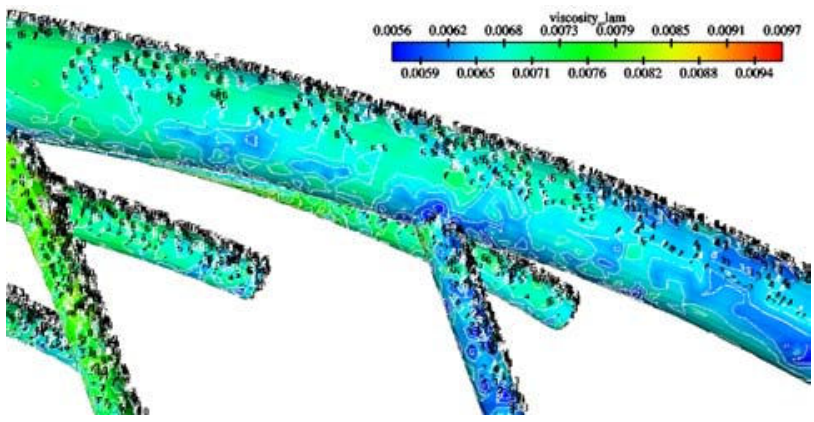

Figure 6. Contour plots of the molecular viscosity $(\mathrm{kg} / \mathrm{m}-\mathrm{s})$ magnitude distribution at the origins of the second (D2) and third diagonal (D3) branches.

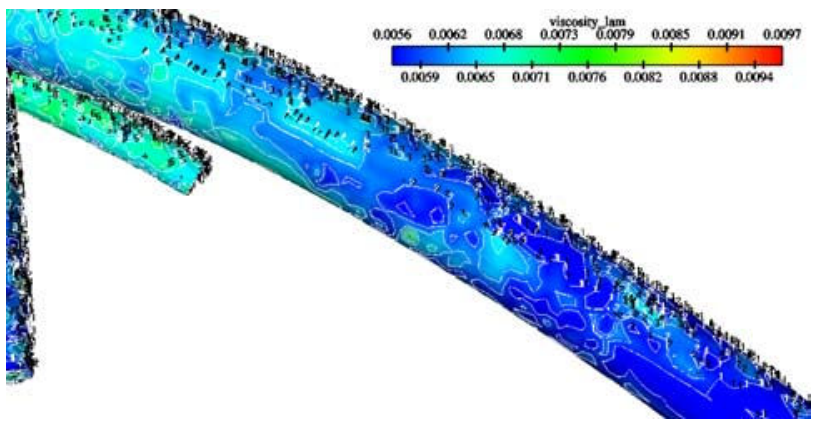

Figure 7. Contour plots of the molecular viscosity (kg/m-s) magnitude distribution at the distal LAD segment origins of the second (D2) and third diagonal (D3) branches.

\section{Discussion}

According to the non-Newtonian behaviour, blood viscosity varies along the course of the vessel. This variation is dependent on the vascular geometry, flow particularities and local composition of blood flow. High viscosity regions appear at the lateral walls of the bifurcations. From the analysis of all contour results, it is evident that there are two distinct flow regions within 
the flow, which possesses high blood viscosity values. The first region is located on the lateral walls of the bifurcations (see Figure 4) and is nearly always characterized by low wall shear values. The second region is located within the central blood flow passage, and occupies large section of the lumen, (see Figure 7). It follows the region of high velocity magnitude. Between these two distinct regions the blood is forced to move with relatively low viscosity values. Since viscosity is a fluid intrinsic resistance to blood flow within vessels, the phenomenon of high viscosity regions in the central part of the lumen moderates the high blood velocity values. However, along the vessel wall, at the regions where low shear stress and low static pressure occur, the effect of blood flow resistance, due to increased blood viscosity, gives rise to the increased contact time between the atherogenic particles of the blood and the endothelium. Preservation molecular viscosity at low level values as well as erythrocytes deformability usually constitute physiologic adjustments in humans in maintaining normal blood flow in coronary arteries

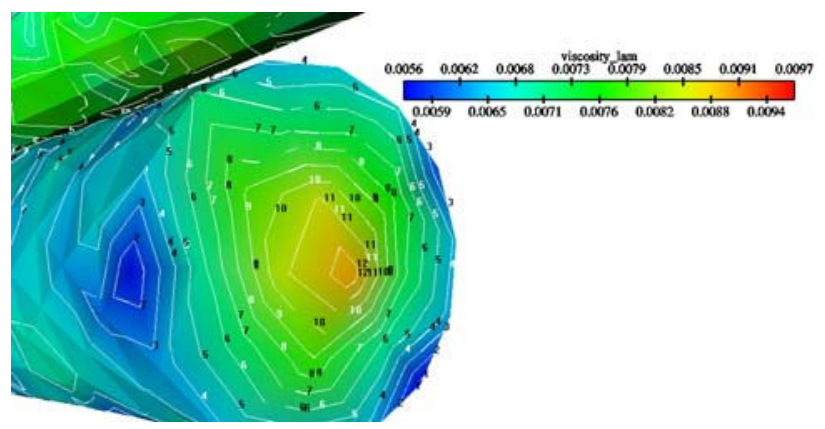

Figure 8. Contour plots of the molecular viscosity $(\mathrm{kg} / \mathrm{m}-\mathrm{s})$ magnitude distribution at a typical diagonal branch (second diagonal).

Preservation molecular viscosity at low level values as well as erythrocytes deformability usually constitute physiologic adjustments in humans in maintaining normal blood flow in coronary arteries. In contrary, it seems reasonable to suggest that flow disturbances combined with increased molecular viscosity contribute to an atherogenic environment.

Furthermore, analysis results indicate that molecular viscosity decreases with decreasing vessel caliber (see Figure 7), at the distal part of the major coronary arteries. Given that atherosclerosis localization is less common at the distal segments compared to the proximal ones, it is also reasonable to deduce that low blood molecular viscosity has a beneficial action efficiently protecting the arterial wall.

\section{Conclusion}

The distribution of high molecular viscosity along the walls is in agreement with the prone to atherosclerosis regions. The effect of blood flow resistance, due to increased blood viscosity, gives rise to increased contact time between the atherogenic particles of the blood and the endothelium, probably, promoting atherosclerosis.

\section{References}

[1] Becker RC. The role of blood viscosity in the development and progression of coronary artery disease. Cleve Clin J Med 1993 Sep-Oct;60(5):353-8.

[2] Danesh J, Collins R, Peto R, Lowe GD. Haematocrit, viscosity, erythrocyte sedimentation rate: meta-analyses of prospective studies of coronary heart disease. Eur Heart J. 2000 Apr;21(7):515-20.

[3] Dodge JT Jr, Brown BG, Bolson EL, Dodge HT. Intrathoracic spatial location of specified coronary segments on the normal human heart. Applications in quantitative arteriography, assessment of regional risk and contraction, and anatomic display. Circulation. 1988 Nov; 78(5 Pt 1): 1167-80.

[4] Dodge JT Jr, Brown BG, Bolson EL, Dodge HT. Lumen diameter of normal human coronary arteries. Influence of age, sex, anatomic variation, and left ventricular hypertrophy or dilation. Circulation 1992 Jul; 86(1): 23246.

[5] Sharma K, Bhat SV. Non-Newtonian rheology of leukemic blood and plasma: are $\mathrm{n}$ and $\mathrm{k}$ parameters of power law model diagnostic? Physiol. Chem. Phys Med NMR 1992; 24(4), 307-12.

[6] Montenegro MR, Eggen DA. Topography of atherosclerosis in the coronary arteries. Lab invest. 1988; 18:586-593.

[7] Halon DA, Sapoznikov D, Lewis BS, Gotsman MS. Localization of lesions in the coronary circulation. Am J Cardiol. 1983; 52:921-926.

Address for correspondence.

George D Giannoglou

Ath. Soulioti 17 Str., Thessaloniki 54642

yan@med.auth.gr 